\title{
Efficiency of Collisionally-Activated Dissociation and 193-nm Photodissociation of Peptide Ions in Fourier Transform Mass Spectrometry
}

\author{
Evan R. Williams, Jorge J. P. Furlong,* and Fred W. McLafferty \\ Chemistry Department, Baker Laboratory, Cornell University, Ithaca, New York, USA
}

\begin{abstract}
For tandem mass spectrometry, the Fourier transform instrument exhibits advantages for the use of collisionally-activated dissociation (CAD). The CAD energy deposited in larger ions can be greatly increased by extending the collision time to as much as $120 \mathrm{~s}$, and the efficiency of trapping and measuring CAD product ions is many times greater than that found for triple-quadrupole or magnetic sector instruments, although the increased pressure from the collision gas is an offsetting disadvantage. A novel system that uses the same laser for photodesorption of ions and their subsequent photodissociation can produce complete dissociation of larger oligopeptide ions and unusually abundant fragment ions. In comparison to CAD, much more internal energy can be deposited in the primary ions using 193-nm photons, sufficient to dissociate peptide ions of $m / z>2000$. Mass spectra closely resembling ion photodissociation spectra can also be obtained by neutral photodissociation (193-nm laser irradiation of the sample) followed by ion photodesorption. (J Am Soc Mass Spectrom 1990, 1, 288-294)
\end{abstract}

$\mathrm{M}$ olecular structure elucidation by tandem mass spectrometry (MS/MS) [1-3] is especially promising for the sequencing of oligopeptides [4-6] and phospholipids [7]. Among the instruments available for such MS/MS applications, the Fourier transform (FT) mass spectrometer [8-15] has the unusual capabilities of multichannel measurement of all stored ions, the use of a single mass analyzer for multiple mass separations $\left(\mathrm{MS}^{n}\right)$ sequential in time, unusually high resolution, and a high upper mass range, prompting this further study of methods for dissociating ions mass-selected by FTMS.

The most common method, collisionally-activated dissociation (CAD) [16, 17], is generally used with pulsed-valve introduction of the target gas because of the unusually low $\left(10^{-9}\right.$ torr) FTMS pressure requirements [18]. Collisionally-activated dissociation has been used with FTMS for oligopeptide sequencing $[10,14]$, resulting in more complete information from the CAD of both positive and negative ions [19]. A disadvantage of $\mathrm{CAD}$ is its serious decrease in both the degree and efficiency of dissociation with increasing mass $[5,6]$. Little useful information is obtained for oligopeptide ions of $\mathrm{m} / z>2500$; this is attributable to a kinetic effect [20] as well as a collisional [21] effect. For specific values of the ion kinetic energy $E_{\mathrm{LAB}}$ and the target gas mass, $m_{g}$, increasing the mass of the pre-

\footnotetext{
* Visiting scientist from the Faculty of Chemistry, National University of San Luis, Argentina.

Address correspondence to Fred W. McLafferty, Department of Chemistry, Cornell University, Ithaca, NY 14853-1301.
}

cursor ion, $m_{\mathrm{p}}$, will reduce the center-of-mass collision energy, $E_{C M}$ (Figure 1) [22]. A further disadvantage of FTMS is that its fixed magnetic field, $B$, and maximum cyclotron radius, $r$, also require that $E_{\mathrm{LAB}}$ be reduced with increasing mass; the combined effect (Figure 1) provides a discouraging prediction for CAD of highmass singly charged ions in FTMS.

The energy deposited in photon absorption is independent of mass. Laser photodissociation of ions is much more effective with stored ions $[11,13,15,22]$ than with high-velocity ions [23], and also with highenergy photons (e.g., $193 \mathrm{~nm}-6.4 \mathrm{eV}$ ), for obtaining sequence information for large peptides $[11,13,15]$. Surface-induced dissociation, pioneered by Mabud et al. [24], is also promising for FTMS [25], and its application to sequencing is discussed in an accompanying paper [26]. Here we will examine the effect of energy deposited under varying conditions by $\mathrm{CAD}$ and photodissociation in FTMS.

\section{Experimental}

A prototype Nicolet FTMS-2000 [14, 25], field strength $2.86 \mathrm{~T}$, was used with a $5.1 \times 5.1 \times 10 \mathrm{~cm}$ ion cell (the commercial dual cell without the conductance limit and with single $10-\mathrm{cm}$ excite and receive plates). Photons $(193 \mathrm{~nm}$ ) from a Lumonics TE-861S excimer (ArF) laser enter the FTMS hollow sample probe through an $\mathrm{MgF}_{2}$ window $21 \mathrm{~cm}$ from the sample end; electrical contact (typically grounded) is made with the probe tip through a CuBe spring clip when the probe is fully inserted. 


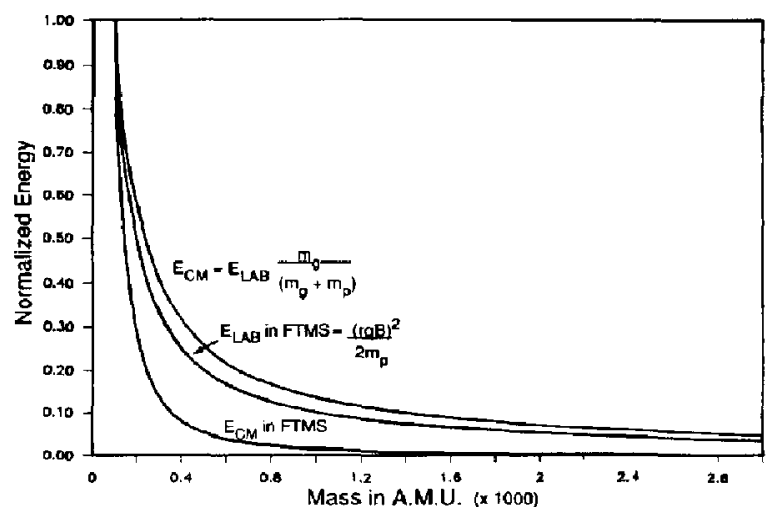

Figure 1. Energy curves as a function of mass for maximum center-of-mass collision energy for a $100-\mathrm{eV}$ parent ion undergoing a collision with Ar (upper, ordinate value $\times 29=$ actual $\mathrm{eV}$ ); maximum translational energy in FTMS (middle, $\times 2700=\mathrm{eV}$ ); and maximum energy available per collision in FTMS (bottom, $\times 771=\mathrm{eV}$ ). Lower two curves are calculated for a singly charged ion with a $2.5-\mathrm{cm}$ radius at a magnetic field strength of $3 \mathrm{~T}$.

Samples, used as obtained commercially, in methanol are deposited by electrospray [14] to a thickness of $1-5 \mathrm{~nm} / \mathrm{cm}^{2}$ on the wires of a $36 \%$ transmittance stainless-steel mesh (400 wires/in.) across the probe end. Rotation of the mesh during spraying improves sample uniformity and reproducibility of spectra from different mesh locations. The laser beam is introduced along the $z$-axis center of the FTMS cell and focused (2-m focal length $\mathrm{MgF}_{2}$ lens) to a $\sim 0.7 \times 3 \mathrm{~mm}$ spot, $\sim 6 \times 10^{6} \mathrm{~W} / \mathrm{cm}^{2}$ per $14 \mathrm{~ns}$-pulse, to desorb ions to be trapped in the cell. $\Lambda$ dditional samples are exposed by rotating the off-center probe.

For photodissociation, the desired ions (without isotopic selectivity) are isolated from those that are laserdesorbed by eliminating unwanted ions with appropriate $\mathrm{rf}$ ejection sweeps. A delay time of $3 \mathrm{~s}$ allows any neutral species that are formed to be pumped away, and the probe is rotated to move the sample mesh out of the laser path. Light from a second laser pulse of the same fluence then passes through the cell to photodissociate the trapped ions. Alignment of the laser is critical, because light striking the cell walls can produce abundant background ions. With gramicidin S, electrostatic ejection of all ions from the cell between laser pulses resulted in complete loss of the sequence ions, indicating that the sole source of these ions was photodissociation of photodesorbed ions. Low-abundance ions formed by multiphoton ionization of ubiquitous background contamination (e.g., $m / z 149$ from phthalates), however, were commonly observed.

For the CAD experiments, similarly selected ions are excited using on-resonance if into cyclotron orbits of a size that produces the greatest degree of fragmentation. Collisions are effected for an interaction time of 3-120 s with Ar introduced via pulsed valves [18] producing a maximum cell pressure of $\sim 5 \times 10^{-6}$ torr, as estimated from the pulse profile [27]. The CAD spectrum is measured at a cell pressure of $\leq 1 \times 10^{-8}$ torr. For gramicidins $S$ and $D$, a background spectrum mea- sured without precursor ion excitation and collision gas introduction was subtracted; that for alamethicin showed no detectable fragment ions below $\mathrm{m} / \mathrm{z} 1800$ and was not subtracted. The collision energy is calculated from the power of the rf excitation but represents a maximum value because the resonant frequency can change slightly with orbital radius [28]. That calculated for alamethicin exceeded the cell dimensions; the value reported corresponds to a radius of $2.3 \mathrm{~cm}$.

\section{Results and Discussion}

\section{Ionization by 193-nm Photodesorption}

To use the same laser for photodesorption and photodissociation with two consecutive laser pulses, the laser beam is directed down the FTMS magnetic axis and focused on a sample mesh immediately in front of the trapping plate at the ion cell entrance. After passing through the cell, the beam diameter is still less than the 4-mm aperture on the back trapping plate because of the long $(2 \mathrm{~m})$ focal length of the lens used for focusing; light striking the trapping plates desorbs background ions (e.g., $\mathrm{Cs}^{+}$from previous laser desorption (LD) experiments with CsI). Backside desorption from a 3- $\mu \mathrm{m}$ Al foil was not successful; similarly, after sample deposition the mesh must still transmit light. Higher transmittance of the sample-loaded mesh generally results in higher fragmentation, indicative of subsequent photodissociation. Nickel, copper, and stainless-steel mesh gave equivalent results. Within these restrictions, positive ion desorption spectra with 193-nm photons were generally similar to those formed with $10.6-\mu \mathrm{m}$ photons $[10,19]$, on the basis of the spectra of gramicidins $\mathrm{S}$ and $\mathrm{D}$, alamethicins, bradykinin, chlorophyll a (Figure 2, top), hydroxycobalamin, vita$\min B_{12}$, substance $P$, and leucine enkephalin. Negative ion spectra (e.g., chlorophyll $a$, Figure 2 bottom) were generally satisfactory, although some were less readily interpretable. Spectra resulting from repeated laser irradiation at the same sample spot are discussed later.

\section{Tandem Mass Spectrometry Structural Information}

For $\sim 170-\mathrm{eV}$ collisions of the $[\mathrm{M}+\mathrm{Na}]^{+}$molecular ion of gramicidin S (MW 1142) for $3 \mathrm{~s}$ with only the laser-desorbed neutrals, the resulting $\mathrm{CAD}$ spectrum (not shown) [29] is similar to those previously reported $[10,14,19]$, except that the high-mass ions are relatively more abundant $(\mathrm{m} / \mathrm{z} 593$ and 1051 of equivalent abundance). All four CAD spectra indicate seven (Phe-Pro-Val-Orn-Leu-Phe-Pro) of the 10 amino acids. With pulsed-valve Ar introduction and $3 \mathrm{~s}$ collision time, the degree of fragmentation is greatly increased (Figure 3, top), so that the largest indicated sequence is -(Leu,Phe)-(Pro, Val)-Orn-Leu-Phe. The pulsed-valve introduction gives a much higher collision gas pressure and produces more collisions and more fragmentation. This has also increased the efficiency and resolution by approximately a factor of 5 over that obtained using static background pressure $[14,29]$. 


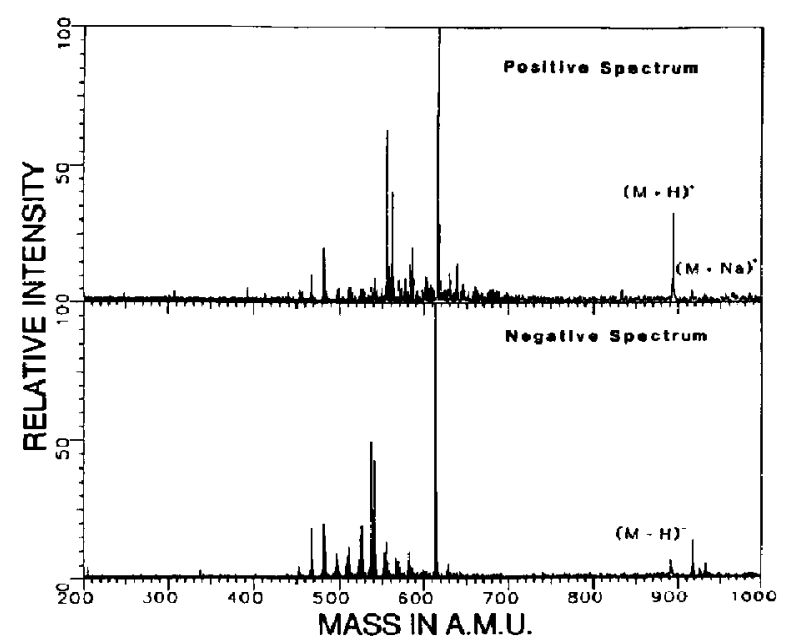

Figure 2. 193-nm photodesorption spectra of chlorophyll $a$; (top) positive ions, (bottom) negative ions.

For ion photodissociation, the 3-s delay after the laser photodesorption pulse is sufficient to remove all photodesorbed neutrals, because with electrostatic removal of the ions there are no fragment ions in the spectrum measured after the second laser pulse. Such photodissociation of photodesorbed gramicidin $S$ ions with 193-nm (6.4-eV) photons (Figure 3, bottom) pro duces more dissociation than all but the pulsed-valve CAD with a 3-s delay, but this still provides the sequence information for seven amino acids. One striking difference between the photodissociation and CAD spectra is the appearance of protonated fragments at $m / z 197$ and 169 corresponding to (Pro-Val) and (Pru$\mathrm{Val}-\mathrm{CO}$ ) in the photodissociation spectrum.
Gramicidin D is a natural mixture of four oligopeptides. Collisionally-activated dissociation of the $75-\mathrm{eV}$ $[\mathrm{M}+\mathrm{Na}]^{+}$ions of its main component (MW 1881) produces fragmentation (Figure 4 , top) similar to the previously reported full CAD spectrum [10]. The sequence information derived is nearly the same, although the signal-to-noise ratio is improved by the present pulsed-valve introduction. Photodissociation of the same $[\mathrm{M}+\mathrm{Na}]^{+}$ions (Figure 4 , bottom) now produces much more extensive fragmentation, consistent with the reduction in $E_{\mathrm{CM}}$ expected for CAD (Figure 1). Dissociation involves the same two major pathways but occurs further down the backbone of the ion. This provides complementary information for sequencing 11 of the 15 amino acids, including an N-terminal Leu not indicated in the CAD spectrum. Other series, such as subsequent loss of $\mathrm{H}_{2} \mathrm{O}$ from the C-terminal series, are also observed and provide useful confirmation of the sequence.

The alamethicin sample is also a mixture, with isomer II differing by substitution of $\mathrm{Aib}$ [-NHC$\left(\mathrm{CH}_{3}\right)_{2} \mathrm{CO}-$ ] for Ala in position 6 of isomer I [30]. Here $\mathrm{CAD}$ for $3 \mathrm{~s}$ of $\sim 100-\mathrm{eV}[\mathrm{M}+\mathrm{Na}]^{+}$ions gave only two or three discernible sequence peaks. However, waiting $120 \mathrm{~s}$ without adding further collision gas for alamethicin II produces (Figure 5) primarily $\mathbf{N}$ terminal fragmentation corresponding to cleavage between the amide bond with additional loss of HCO for the amino acids $1-13$, providing the sequence for this part of the molecule. For alamethicin I, this same series is observed for amino acids $4-13$ but is shifted by -14 $u$ for fragments with $m / z>460.2$, indicating the substitution of Aib for Ala at position 6 . Somewhat more extensive fragmentation is observed in the 6.4-eV photodissociation spectrum of alamethicin I and II (cumbined, Figure 6, bottom) but with the same cleavages

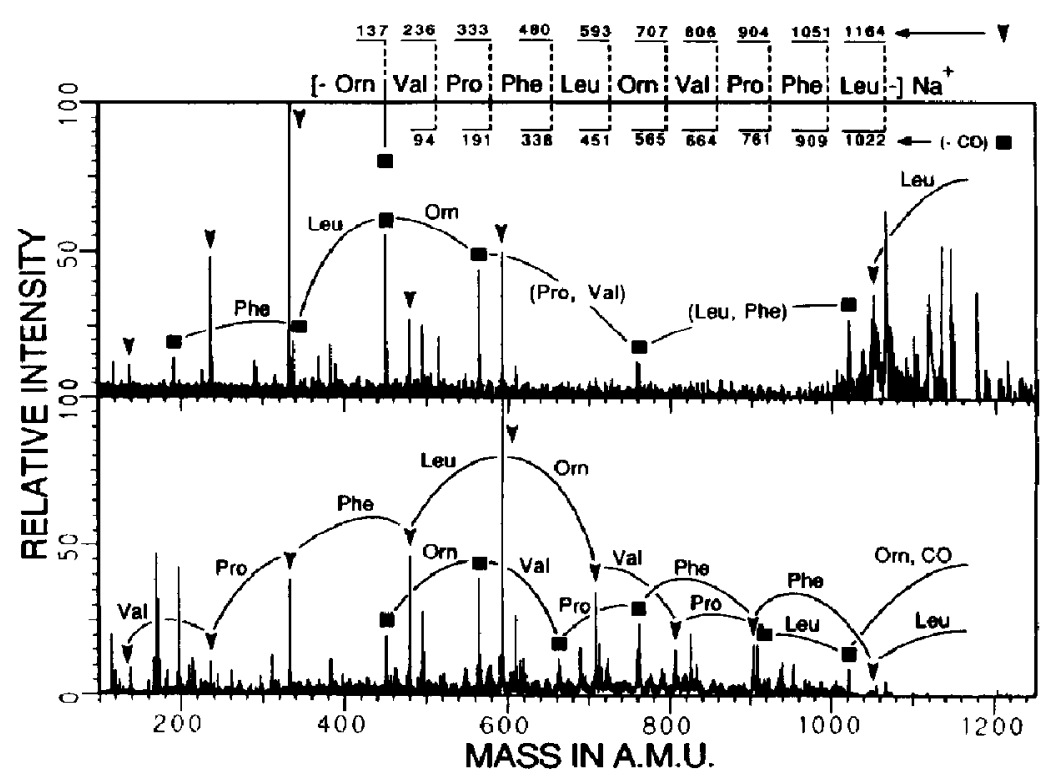

Figure 3. MS-II spectra of $[\mathrm{M}+\mathrm{Na}]^{+}$ion of gramicidin $\mathrm{S}$ formed by a single laser shot and dissociated by 170-eV CAD (top) and 193-nm photodissociation (bottom). ( $)$ Sequence peaks corresponding to initial cleavage between Leu and Orn; (a) iritial cleavage between Orn and $\mathrm{Val}$ with subsequent loss of $\mathrm{CO}$. 


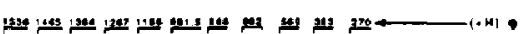

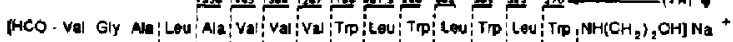

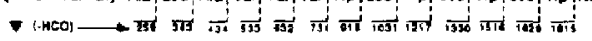
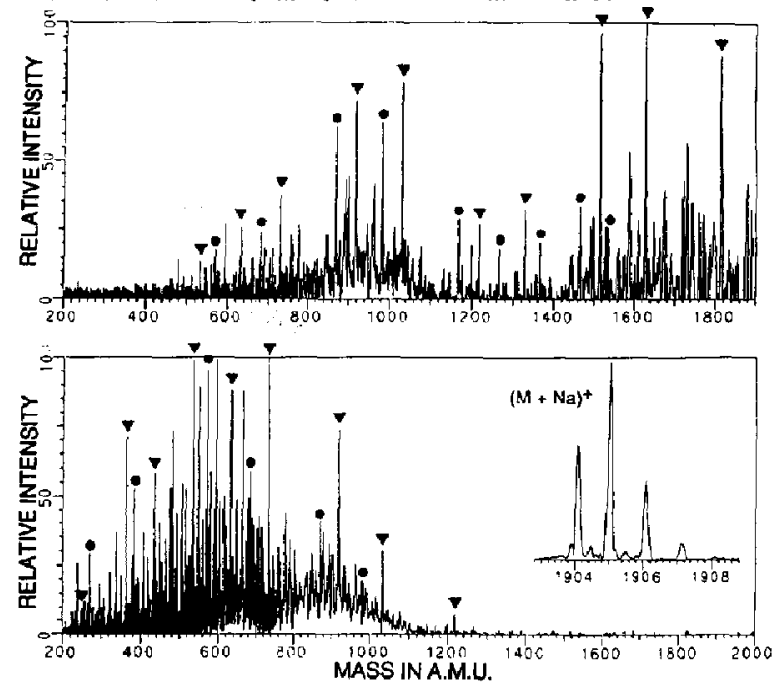

Figure 4. Dissociation spectra of $[\mathrm{M}+\mathrm{Na}]^{+}$ion of gramicidin $\mathrm{D}$ by $75-\mathrm{eV}$ CAD (25 signal-averaged scans, top) and 193- $\mathrm{nm}$ photodissociation (one scan, bottom); medium-resolution (15 K, FWHH) spectrum of molecular ion region inset. $(\bullet, \nabla)$ sequence peaks corresponding to $\mathrm{C}$ - and $\mathrm{N}$ - terminal fragmentation, respectively.

after amino acids $3-13$ and 15 for the two series representing both components. An additional series for the initial loss of $\mathrm{H}$, not $\mathrm{HCO}$, also appears $28 \mathrm{u}$ higher (note the peak quartets spaced $14 \mathbf{u}$ apart) and is often of higher abundance for several of the fragments, providing useful confirmation of the sequence. A 14-u difference is observed for this series above $m / z 460.2$, again indicating the position of substitution of $\mathrm{Aib}$ for Ala at position 6 .

\section{Collisionally-Activated Dissociation Efficiency}

For this photodesorption, shat-to-shot reproducibility of laser power and sample inhomogeneity result in uncertainty of $\sim 30 \%$ in primary ion production. Within this restriction, for gramicidin $S$ the total product ion abundance from pulsed-valve CAD is $\sim 25 \%$ of that of the precursor ions without $\mathrm{CAD}$, a significant improvement over the few percent obtained by static CAD [14]. With gramicidin $D$ and alamethicin, the pulsed-valve CAD efficiency decreases to a few percent. More accurate efficiencies could be determined for alamethicin by using the $[\mathrm{M}+\mathrm{Na}]^{+}$ions of the mixture component not dissociated as an abundance reference; increasing the reaction time from $3 \mathrm{~s}$ to $120 \mathrm{~s}$ for alamethicin increases the CAD yield from $\sim 2 \%$ to $\sim 15 \%$. (Although some of this increase could be due to z-axis relaxation [31], this would not account for the observed increase in the relative abundance of the fragment ions.) Much of this decrease could arise from poor detection of daughter ions from CAD of higher mass species [32]. When a precursor ion undergoes a dissociative collision, the fragment will travel with the same velocity as the parent and will have energy corresponding to a ratio of the daughter/parent masses. Low-mass fragments will have substantially less kinetic energy than the precursors and thus much smaller orbits; orbits that originate from excited precursors will be centered near the cell
Figure 5. CAD spectra of $[\mathbf{M}+\mathrm{Na}]^{+}$ ion of alamethicin I (top), alamethicin II (middle), and combined photodissociation of I and II (bottom). ( $\mathbf{T}) \mathrm{N}$ Terminal fragmentation (alamethicin I labeled in photodissociation spectrum).

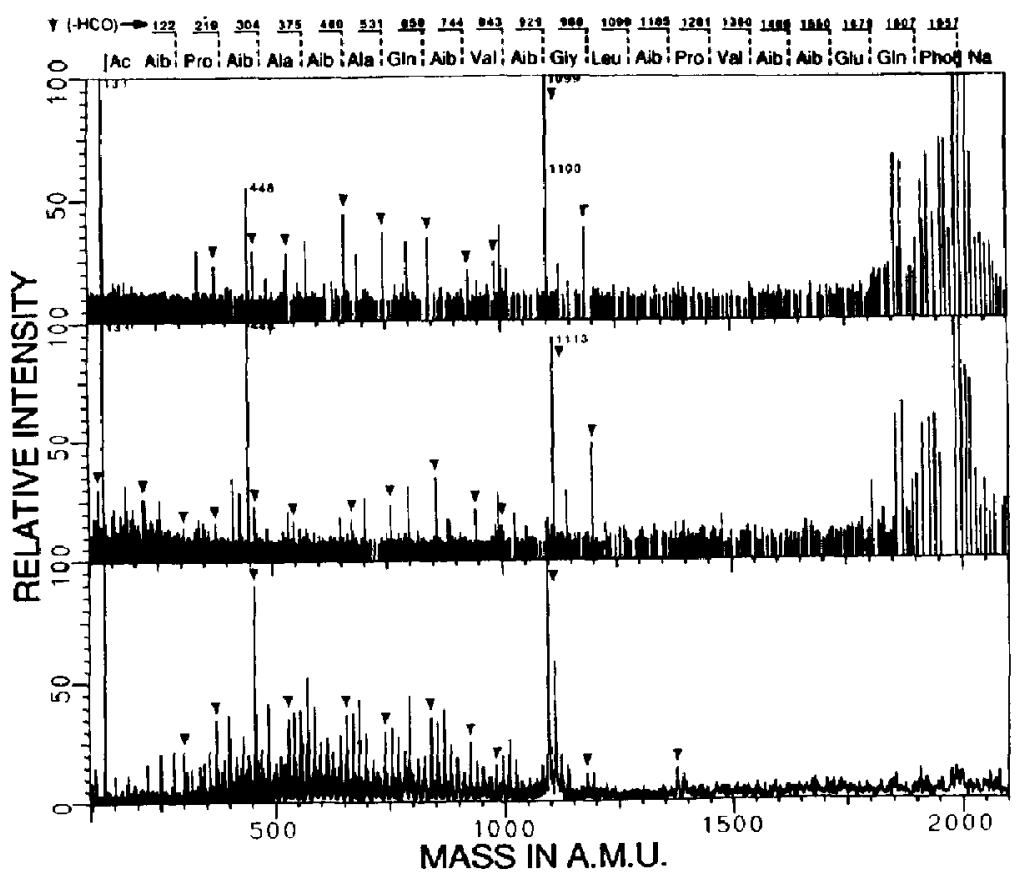




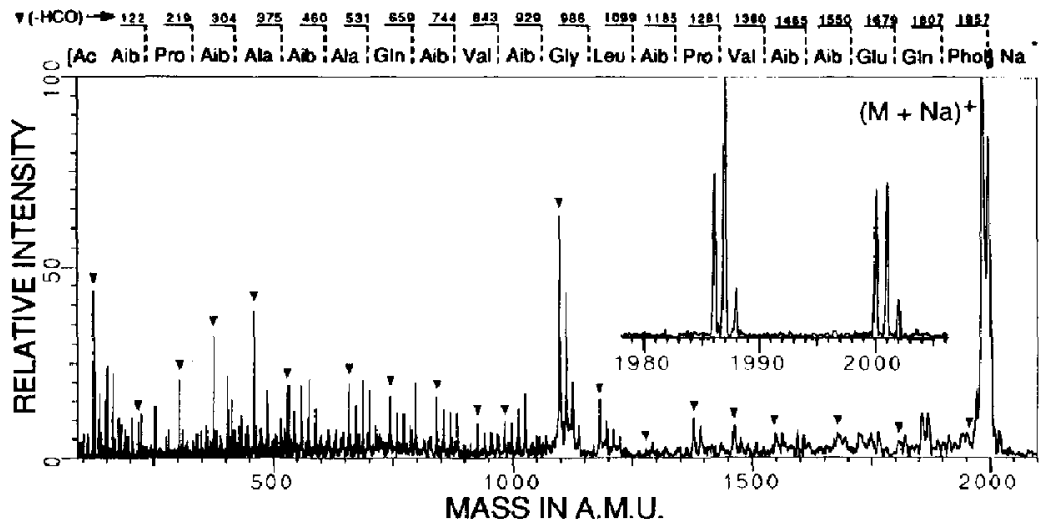

Figure 6. Laser desorption spectrum of alamethicin produced by the fifth laser shot on the same sample spot. ( $\nabla) \mathrm{N}$ Terminal sequence peaks for alamethicin I. Medium-resolution (11 K) spectrum of molecular ion region inset. excite and receive plates. The subsequent detection if pulse will tend to excite these fragment ions to orbits that intercept the cell plates, reducing their detection efficiency. This necessitates reducing the power of the dissociation and/or fragment detection pulses, which also lowers the sensitivity.

\section{Photodissociation Efficiency}

Translational excitation is not necessary for photodissociation, so the surprising complete disappearance of the selected precursor ions found possible for all samples from one laser pulse indicates a dissociation efficiency of nearly $100 \%$; we see no obvious alternative mechanisms for appreciable loss of precursor ions. This high efficicncy indicates that those photodesorbed high-mass ions that are trapped have less than $0.05 \mathrm{eV}$ of kinetic energy in the $x y$ plane, so that these ions have virtually the same physical $x y$ orientation as the second laser pulse. This highly directed expansion is consistent with the previous observation of angular distributions following photodesorption of $\cos ^{4} \theta$ to $\cos ^{10} \theta$ from near-surface gas-phase collisions ("Knudsen layer formation") [33]. In addition, separate studies [34] show unusually high cross sections (eight times that of a same-mass CsI cluster ion) for collisional relaxation of oligopeptide ions back to the center of the ion cell. Energy-selective trapping may also be a contributing factor. Photodissociation has the further advantage that fragments are produced in the center of the cell with little excess kinetic energy and should have detection efficiencies comparable to those of the same fragment ions formed by laser desorption. With gramicidin D, the sum of the product ion abundance is approximately three times that of the precursor ion without photodissociation, indicating a correspondingly higher detection efficiency for these lowmass ions. This is consistent with the observation that high-mass peptide ions have significantly shorter transients than CsI clusters of comparable mass [34, 35] and thus a correspondingly lower detection efficiency. Other factors, such as less efficient coherent excitation at high mass, may also be contributing factors [32].
The dissuciation efficiency can be reduced, however, if the precursor ions are translationally excited by the rf sweeps used for their isolation. For gramicidin $S$, a fragment ejection sweep from $m / z 109$ to $m / z 1020$ reduces the efficiency for dissociation of the $[\mathrm{M}+\mathrm{Na}]^{+}$species to $\sim 45 \%$ (measured by reduction in parent ion abundance). For alamethicin, ejection of fragments from $m / z 110$ to $m / z 1555$ had no effect on the efficiency, but ejection of the $[\mathrm{M}+\mathrm{Na}]^{+}$ion of either alamethicin $I$ or $\amalg$ resulted in virtually no fragmentation of the other $[\mathrm{M}+\mathrm{Na}]^{+}$ion in the subsequent photodissociation attempt. Some net excitation from the ejection pulse apparently is absorbed by the parent ions, bringing them out of the path of the second laser shot. This problem should be significantly reduced by using SWIFT excitation [36, 37], which has no net dc offset and is capable of producing sharp excitation windows.

\section{An Alternative Laser Dissociation Technique}

For photodesorption ionization, additional laser shots at the same spot of the sample produce spectra with increasing fragmentation at the expense of the molecular ion, with the fragmentation closely resembling (Figure 6) that obtained by photodissociation. For alamethicin, the same N-terminal fragmentation series formed by photodissociation is obtained, but this now provides the sequence of 19 of the 20 amino acids. Sample thickness has a large effect; this was $\sim 5 \mathrm{nmol} / \mathrm{cm}^{2}$ $\left(\sim 10^{-10} \mathrm{~mol}\right.$ in the laser spot) for the spectrum of Figure 6 . The same thickness gives few fragments with the first laser shot (Figure 7). 'The second and third shots on the same spot produce nearly identical spectra, but fragmentation starts to increase with subsequent shots. However, if the sample thickness is reduced to $\sim \mathbf{2 0 \%}$ of this value, the first laser shot shows substantially more fragmentation (Figure 7), with much more extensive fragmentation from a second shot on the same spot. With a third shot, primarily low-mass ( $m / z<500$ ) fragment ions are formed. As sample is consumed, the visual transmittance through the sam- 


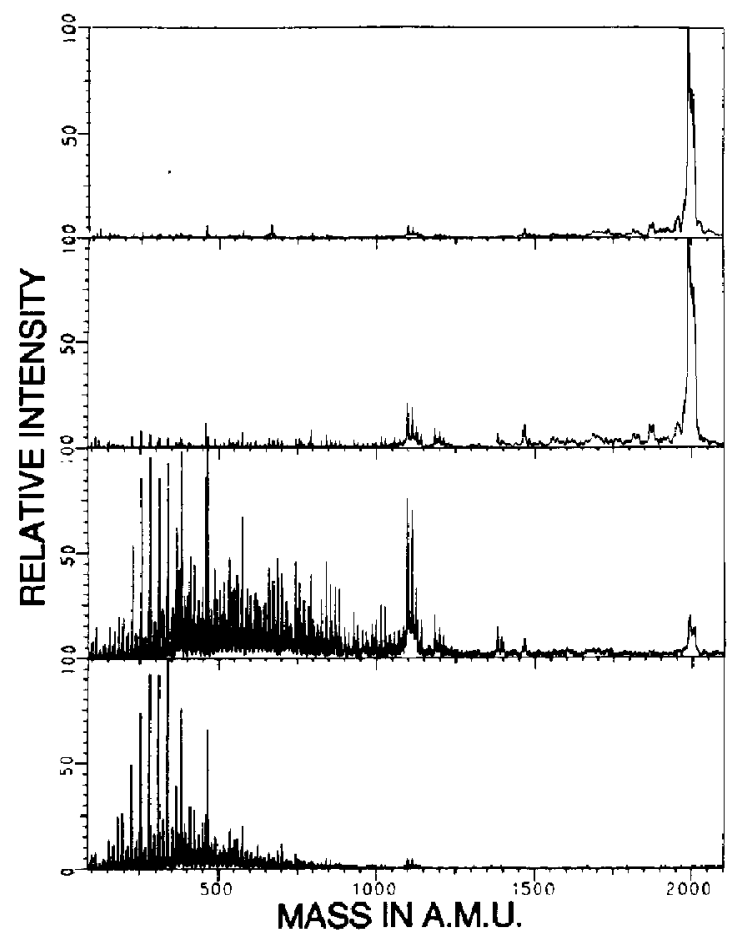

Figure 7. Laser desorption spectra of alamethicin formed by a single shot on a "thick" sample (top), and first, second, and third shot spectra (upper middle to bottom) on the same sample spot for a "thin" sample.

ple increases; so that photodissociation can also occur; however, the extent of dissociation of Figure 7 (bottom) can be due only in minor part to photodissociation. For thin samples, the molecules that absorb the laser photons have a higher probability of being those desorbed as ions. Conversely, thicker samples may make possible a soft shock-wave desorption mechanism [38].

\section{Conclusions}

To offset the decreasing efficiency of CAD in FTMS for peptides of increasing size, the number of collisions can be increased by using a pulsed valve and by increasing the time for collisions and dissociation to occur. Photodissociation of larger oligopeptides with 193-nm photons, for which the energy deposition is independent of mass, exhibits the unusual advantage of nearly $100 \%$ dissociation efficiency when the precursor ions can be confined to the laser beam path, in this case by using the same laser for photodesorption. Repeated laser shots on the same sample spot produce increasing fragmentation in the photodesorption spectra, similar to that obtained by photodissociation. For alamethicin, this technique produces sequence ions for 19 of $\mathbf{2 0}$ amino acids, far more than that from photodissociation alone or CAD.

\section{Acknowledgments}

We are grateful to J. A. Loo, D. F. Hunt, and M. L. Gross for helpful discussions. Generous financial support for this research was provided by the National Institutes of Health, grant GM16609 , with instrumentation by them and the National Science Foundation, grant CHF-8303340, and sponsorship of an ACS Analytical Division Fellowship for E. R. W. by Perkin-Elmer and a postdoctoral fellowship for J. J. P. F. by CONICET, Argentina.

\section{References}

1. McLafferty, F. W., Ed. Tandem Mass Spectrometry; Wiley: New York, 1983

2. Cheng, M. T.; Barbalas, M. P.; Pegues, R. F.; McLafferty, F. W. J. Am. Chem. Soc. 1983, 105, 1510-1513.

3. Levsen, K. In Tandem Mass Spectrometry; McLafferty, F. W., Ed.; Wiley: New York, 1983; pp 41-66.

4. Wipf, H.-K.; Irving, P.; McCamish, M.; Venkataraghavan, R.; McLafferty, F. W. J. Am. Chem. Soc. 1973, 95, 3369-3375.

5. Biemann, K.; Martin, S. A. Mass Spectrom. Rev. 1987, 6, 1-76.

6. Hunt, D. F.; Shabanowitz, I.; Yates, J. R., III; Griffin, P. R.; Zhu, N. Z. In Methods in Protein Sequence Analysis; Wittmann-Liebold, B., Ed.; Springer Verlag: New York, 1989; pp 183-190.

7. Jensen, N. J.; Gross, M. L. Mass Spectrom. Rev. 1988, J, 41-69.

8. Comisarow, M. B.; Marshall, A. G. Chem. Phys. Lett. 1974, 25, 282-283.

9. Laude, D. A.; Johlman, C. L.; Brown, R. S.; Weil, D. A.; Wilkins, C. L. Mass Spectrom. Rev. 1986, 58, 483-485.

10. Cody, R. B.; Amster, I. J.; McLafferty, F. W. Proc. Natl. Acad. Sci. U.S.A. 1985, 82, 6367-6370.

11. Bowers, W. D.; Delbert, S.-S.; Mclver, R. T. Anal. Chem. 1986, 58, 969-972.

12. Amster, I. J.; McLafferty, F. W.; Castro, M. W.; Russell, D. H.; Cody, R. B.; Ghaderi, S. Anal. Chem. 1986, 58, 483-485.

13. Hunt, D. F.; Shabanowitz, J.; Yates, J. R., III. J. Chem. Soc. Chem. Commun. 1987, 548-550.

14. Amster, J. I.; Loo, J. A.; Furlong, J. J. P.; McLafferty, F. W. Anal. Chem. 1987, 59, 313-317.

15. Hunt, D. F.; Shabanowitz, J.; Yates, J. R., III; Griffin, P. R.; Zhu, N. Z. Anal. Chim. Acta 1989, 225, 1-10.

16. McLafferty, F. W.; Bente, P. F., III; Kornfeld, R.; Tsai, S.-C.; Howe, I. I. Am. Chem. Soc. 1973, 95, 2120-2129.

17. Cody, R. B.; Freiser, B. S. Anal. Chem. 1982, 54, 1431-1433.

18. Carlin, T. J.; Freiser, B. S. Antl. Chem. 1982, 55, 571-574.

19. Yang, L.-C.; Wilkins, C. L. Org. Mass Spectrom. 1989, 24, 409-414.

20. Sheil, M. M.; Derrick, P. J. Org. Mass Spectrom. 1988, 23, $429-435$.

21. Bricker, D. L.; Russell, D. H. J. Am. Chem. Soc. 1986, 108, 6174-6179.

22. Nuwaysir, L. M.; Wilkins, C. L. Anal. Chem. 1989, 61, 689-694.

23. Tecklenburg, R. E., Jr.; Miller, M. N.; Russell, D. H. J. Am. Chem. Soc. 1989, 111, 1161-1171.

24. Mabud, M. A.; DeKrey, M. J.; Cooks, R. G. Int. J. Mass Spectrom. Ion Processes 1985, 67, 285-294.

25. McLafferty, F. W.; Amster, I. J.; Furlong, J. J. P.; Loo, J. A.; Wang, B. H.; Williams, E. R. In Fouriet Transform Mass Spectrometry; Buchanan, M. V., Ed.; ACS Symp. Ser. 359; American Chemical Society, Washington, DC, 1987.

26. Williams, E. R.; Henry, K. D.; McLafferty, F. W.; Shabanowitz, J.; Hunt, D. F. J. Am. Soc. Mass Spectrom. 1990, 1.

27. Cody, R. B. Anal. Chem. 1988, 60, 917-923.

28. Wang, M.; Marshall, A. G. Anal. Chem. 1989, 61, 1288-1293.

29. Loo, J. A.; Williams, E. R.; Furlong, J. J. P.; Wang, B. H.; McLafferty, F. W. In Proceedings of the 35th ASMS Conference 
on Mass Spectrometry and Allied Topics; Denver, CO, May 1987, pp 727-728.

30. Pandy, R. C.; Cook, J. C., Jr.; Rinehart, K. L., Jr. I. Am. Chem. Soc. 1977, 99, 8469.

31. Rempel, D. L.; Huang, S. K.; Gross, M. L. Int. J. Mass Spectram. Ion Processes 1986, 70, 163-184.

32. Hanson, C. D.; Castro, M. E.; Russell, D. H. Anal. Chem. $1989,61,2130-2136$.

33. Kelly. R.; Dreyfus, R. W. Nucl. Instr. Methods Phys. Res. 1988, B32, 341-348.

34. Williams, E. R.; Herry, K. D.; McLafferty, F. W. J. Am. Chem. Soc., accepted.
35. Hanson, C. D.; Castro, M. E.; Russell, D. H.; Hunt, D. F.; Shabanowitz, I. In Fourier Transform Mass Spectrometry; Buchanan, M. V., Ed.; ACS Symp. Ser. 359; American Chemical Society, Washington, DC, 1987; pp 100-115.

36. Chen, L.; Wang, T. C.; Ricca, T. L.; Marshall, A. G. Anal. Chem. 1987, 59, 449-454.

37. Marshall, A. G.; Wang, T.-C. L.; Chen, L.; Ricca, T. L. In Fourier Transform Mass Spectrometry; Buchanan, M. V., Ed.; ACS Symp. Ser. 359; American Chemical Society, Washington, DC, 1987; pp 21-33.

38. Vertes, A.; Juhasz, P.; De Wolf, M.; Gijbels, R. Scanning Microsc. 1988, 2, 1853-1877. 